\title{
Improving the Evaluation of Diffuse Infiltrative Lung Diseases by Means of Mutual Education: Difficult Task, but Worth a Try
}

\author{
Maurizio Zompatori ${ }^{a}$ Tiziana Laporta ${ }^{b}$ \\ ${ }^{\text {a } D e p a r t m e n t ~ o f ~ R a d i o l o g y, ~ U n i v e r s i t y ~ H o s p i t a l ~ o f ~ P a r m a, ~ U n i v e r s i t y ~ o f ~ P a r m a, ~ a n d ~}{ }^{\text {b } T S R M, ~}$ \\ Department of Radiology, University Hospital of Bologna, S. Orsola-Malpighi Hospital, Bologna, Italy
}

In this issue of Respiration, Diette et al. [1] present the results of an interesting analysis, examining whether US pulmonologists are prepared and willing to accept a highresolution computerized tomography (HRCT) diagnosis of usual interstitial pneumonitis/idiopathic pulmonary fibrosis (UIP/IPF), instead of a lung biopsy.

This idea makes sense because transbronchial lung biopsy presents obvious and important limitations in this field, whereas surgical biopsy is not always feasible and is associated with not trivial morbidity and mortality rates [2]. Furthermore, diffuse lung diseases (DLDs) often present regional heterogeneities which represent problems for the reading pathologist, especially when multiple biopsies of different lobes cannot be performed. For example, according to different authors [3-5], discordant findings in multiple biopsies can be found in up to $26 \%$ of patients affected by idiopathic interstitial pneumonias (IIPs). In addition to the sampling error, histology also presents difficulties related to inter-observer agreement.

Nicholson et al. [6] reported that in 18\% of the biopsy specimens the diagnosis was given by pathologists with low confidence; the largest variation (over 50\%) was found in the differential diagnosis between UIP/IPF and nonspecific interstitial pneumonia (NSIP). Otherwise, providing clinical and radiological information to pathologists can lead to a substantial change in the pathological diagnosis in $20 \%$ of IIPs. For these reasons, a reliable noninvasive diagnosis of DLDs should be welcomed by all pulmonologists across the pond. We assume that the results of this study can also be reasonably applicable to specialists outside the US.

A secondary aim of the study was to examine the consistency of physicians' practices with the current evidence-based guidelines [7]. The information obtained was significantly more extensive because a list of the 16 more common subacute or chronic diffuse conditions was also added to the questionnaire. The participating pulmonologists were asked to indicate how frequently in their practice they were willing to rely on the HRCT diagnosis of these conditions to begin a treatment, assuming that the HRCT diagnosis, made by an experienced radiologist, was concordant with the clinical presentation.

$23 \%$ of responders indicated that HRCT always or frequently obviated the tissue diagnosis; $53 \%$ that HRCT can sometimes do so, and 24\% seldom or never. Most pulmonologists do not believe that HRCT is sufficiently specific to avoid biopsy, with a notable exception of UIP/ IPF (2/3 of responders), coniosis and bronchiectasis. Thus, regarding UIP/IPF, the prevalent opinion was consistent with the more recent guidelines [7] stating that surgical biopsy is indicated only for patients without a typical HRCT and clinical presentation, mainly to differentiate between UIP and NSIP.

As a matter of fact, it is well known that the accuracy of a confident diagnosis of UIP on HRCT is upwards to $90 \%$ for experienced observers [8]. State of the art CT also allows a precise score of honeycombing and consistent

\section{KARGER \\ Fax +4161306 1234 E-Mail karger@karger.ch} www.karger.com
(C) 2005 S. Karger AG, Basel 0025-7931/05/0722-0127\$22.00/0

Accessible online at: www.karger.com/res
Prof. Maurizio Zompatori, MD

Department of Radiology, University Hospital of Parma

Via Gramsci 14

IT-43100 Parma (Italy)

Tel. +390521 703219, Fax +390521 936352, E-Mail maurizio.zompatori@unipr.it 
serial follow-up examinations to be made, which are both useful in predicting the prognosis [9].

Biopsy is also considered appropriate in symptomatic subjects with a clinical suspicion of DLDs when HRCT is normal, because the false-negative rate of this imaging method is about $10 \%$ for the entire group of DLDs and false-negative cases can even be found, albeit more rarely, in histologically proven UIP/IPF [10-12].

Notice that the article of Diette et al. [1] refers to chronic conditions, mainly characterized by interstitial or cystic patterns on HRCT. Admittedly, acute diseases or diseases presenting with a predominant ground glass opacity or consolidative pattern can be more difficult to characterize precisely using HRCT $[2,13]$. The HRCT diagnosis of the remaining 13 subacute or chronic diseases, including lymphangioleiomyomatosis (LAM) and Langerhans cell histiocytosis (LCH), was not deemed an acceptable substitute for biopsy.

Pulmonologists poorly trained to interpret $\mathrm{CT}$ images were significantly more likely to believe that $\mathrm{CT}$ is seldom or never able to suggest the correct diagnosis, versus those referring 5 or more patients per month for HRCT studies.

The reticence to accept a HRCT diagnosis of the other entities included in the questionnaire, such as LAM or $\mathrm{LCH}$, in lieu of biopsy was somewhat unexpected. In fact, HRCT is significantly more accurate than chest X-ray in the detection and characterization of DLDs [10, 11, 14], provided that the diagnostic approach also encompasses the time factor and the clinical context. HRCT diagnosis is highly reliable in a substantial array of common and uncommon DLDs [9, 13-16].

Aziz et al. [17] demonstrated that there is good agreement between radiologists for HRCT evaluation of a number of DLDs in which the findings are rather typical, in the appropriate clinical context, although a lower confidence and agreement is typically found in the diagnosis of NSIP versus UIP/IPF.

The likely reason of the physicians' reticence reported by Diette et al. [1] is that certain diseases are relatively rare and most pulmonologists are not familiar enough with them. Therefore, further educational efforts should be made to increase the awareness of HRCT strengths and limitations in this field, helping to obviate unnecessary biopsies, at least in typical cases.

$\mathrm{We}$, the radiologists, must educate pulmonologists and, of course, be educated by them, in a continuous interplay. This need seems somewhat at odds with the trend of contemporary radiology towards advanced technology (PACS, CAD programs and so on), separating radiologists more and more from other specialists by site and time in a sort of involuntary professional seclusion. However, the effort is definitely worth a try, because 'a decision team is always better than a decision tree' [18].

On the top of all that, we now acknowledge that, in the realm of DLDs and particularly in the diagnosis of IIPs, a pathologic gold standard simply does not exist. Fortunately, we are left with a useful silver standard, consisting of a dynamic, daily interaction between clinicians, radiologists and pathologists.

\section{References}

1 Diette GB, Scatarige JC, Haponik EF, Merriman B, Fishman EK: Do high-resolution CT findings of usual interstitial pneumonitis obviate lung biopsy? Views of pulmonologists. Respiration 2005;72:134-141.

2 Poletti V, Chilosi M, Olivieri D: Diagnostic invasive procedures in diffuse infiltrative lung diseases. Respiration 2004;71:107-119.

3 Flaherty K, Travis W, Colby T, et al: Histopathologic variability in usual and non-specific interstitial pneumonias. Am J Respir Crit Care Med 2001;164:1722-1727.

4 Flaherty K, Thwaite E, Kazerooni E, et al: Radiological versus histological diagnosis in UIP and NSIP. Survival implications. Thorax 2003; 58:143-148.

5 Monaghan H, Wells A, Colby T, et al: Prognostic implications of histologic patterns in multiple surgical biopsies from patients with idiopathic interstitial pneumonias. Chest 2004; 125:359-360

6 Nicholson A, Addis B, Bharucha H, et al: Interobserver variation between pathologists in diffuse parenchymal disease. Thorax 2004;59: 500-505.
7 American Thoracic Society/European Respiratory Society International Multidisciplinary Consensus Classification of the Idiopathic Interstitial Pneumonias. This joint statement of the American Thoracic Society (ATS), and the European Respiratory Society (ERS) was adopted by the ATS board of directors, June 2001 and by the ERS Executive Committee, June 2001. Am J Respir Crit Care Med 2002;165: 277-304.

8 Ellis S, Hansell D: Idiopathic interstitial pneumonias. Imaging-pathologic correlation. Eur Radiol 2002; 12:610-626.

9 Hansell D: High resolution CT of diffuse lung disease. Value and limitations. Radiol Clin North Am 2001;39:1091-1113.

10 Nishimura K, Izumi T, Kitaichi M, et al: The diagnostic accuracy of HRCT in diffuse infiltrative lung diseases. Chest 1993;104:1149-1155.

11 Zompatori M, Bnà C, Poletti V, et al: Diagnostic imaging of diffuse infiltrative diseases of the lung. Respiration 2004;71:4-19.
12 Olivieri D, Poletti V, Zompatori M: Interstitial lung diseases. Introduction. Respiration 2004; $71: 3$.

13 Ryu J, Olson E, Midthun D, Swensen S: Diagnostic approach to the patient with diffuse lung disease. Mayo Clin Proc 2002;77:1221-1227.

14 Schaefer-Prokop C, Prokop M, Fleischman D, Herold $\mathrm{H}$ : High resolution CT of diffuse interstitial lung disease. Key findings in common disorders. Eur Radiol 2001;11:373-392.

15 Padley S, Hansell D, Flower C, Jennings P: Comparative accuracy of HRCT and chest radiography in the diagnosis of chronic diffuse infiltrative lung disease. Clin Radiol 1991;44: 222-226.

16 Hartman T: CT of cystic diseases of the lung. Radiol Clin North Am 2001;39:1231-1234.

17 Aziz Z, Wells A, Hansell D, et al: HRCT diagnosis of diffuse parenchymal lung disease. Interobserver variation. Thorax 2004;59:506511.

18 Athanasoulis C, Lee A: Algorithms in radiology and medical imaging. Radiology 1987;164: $565-567$. 